\title{
Global Control of Multi-Machine Power Systems for Transient Stability Enhancement
}

\author{
G. H. Zhang, Y. Wang and D. J. Hill
}

\begin{abstract}
This paper presents a global control strategy for multi-machine power systems to enhance transient stability. The global control system models are proposed to represent the power systems with abrupt structure changes. Multi-machine power systems are first represented by interconnected subsystems with uncertainties and nonlinearities. Then each subsystem is decomposed into four nonlinear partial systems according to switching actions of breakers under a severe disturbance. The partial feedback controllers are respectively designed for partial models and the decentralized controllers for each subsystem are obtained through membership functions. Finally, the global controller is the summation of all the control actions from every subsystem. A three-machine power system is used as an example to demonstrate the effectiveness of the proposed global control strategy for multi-machine power systems.
\end{abstract}

\section{INTRODUCTION}

$\mathrm{T}$ he development of power systems has increasingly inspired the strict controller performance from a global operation. As the deregulation of power industry and environment concerns advance recently, the transmission lines are expected to operate at maximum capacity close to thermal limits without expansion of the existing transmission and generation infrastructure. Intuitively, system behavior will be more dependent on nonlinear characteristics and thus more complex. Consequently, control based capability and controller interactions play an important role for reliable and economical operation of power system, especially when it is subjected to disturbances.

In the recent literature, the nonlinearities, uncertainties, and control systems interactions become highlighted issues to be solved in large-scale power system modeling and control. Adaptive nonlinear technique, robust control theory, neural network, fuzzy logic, genetic algorithm and decentralized control technique are utilized to design controllers for transient stability improvement [1]-[6]. Multi-machine power systems were approximated by linear models with certain uncertainties and nonlinearities were bounded by polynomials or represented by neural network. As the power system is a real life system and operates in wide operating region, the dynamic characteristic of large-scale power systems may not

Y. Wang is with School of Electrical and Electronic Engineering, Nanyang Technological University, Singapore 639798. (e-mail: eyywang@ntu.edu.sg).

D. J. Hill and G. H. Zhang are with Department of Information Engineering, Building 115, Research School of Information Sciences and Engineering, The Australian National University, Canberra, Australia, ACT 0200. (e-mail: David.Hill@anu.edu.au) be accurately described by a single nonlinear model when it is affected by the disturbance, especially the derivatives of disturbance. Global control scheme is proposed to solve those practical problems ([7] and [8]). It combines the qualitative and quantitative knowledge through some hierarchy to coordinate various control actions, which deal with all the anticipated problems and operating conditions. It has been successively applied to the example power systems in our previous work ([9] and [10]).

This paper is aimed to extend global control strategy to the large-scale interconnected power systems in consideration of structure changes resulted from the large disturbances. The basic idea of global control of multi-machine power system is to divide the power system into the subsystems with interconnections first. Then each subsystem is decomposed into four nonlinear partial systems according to switching actions of breakers under a severe disturbance. The partial feedback controllers are respectively designed for partial models and the decentralized controllers for each subsystem are obtained through membership functions. Finally, the global controller is the summation of all the control actions from every subsystem to guarantee the stabilization of the interconnected nonlinear systems. Since we exclude the switching moment of breakers during the transient analysis, uncertain and nonlinear bounds of each partial model can be easily obtained and the global stability of multi-machine power system can be guaranteed.

The rest of the paper is organized as follows. Section II gives the multi-machine power systems model after using direct feedback linearization. The design of the global controller for multi-machine power systems is proposed in Section III. This control scheme is implemented in a three-machine power system and simulation results are provided to demonstrate the performance of the proposed global control approach in Section IV. Conclusions are drawn in Section V.

\section{Multi-Machine POWER SYSTEM MOdel}

The classical power system model is used for a multi-machine system according to certain assumptions. Thus the $N$ machine system is reduced to an electrical network with $N$ internal machine buses. The $i$ th $(i=1,2 \ldots N)$ machine in the lossless power system with generator excitation control can be described as follow ([11] and [12]): (The notation for the system model is given in [12])

Mechanical Equation: 
$\Delta \dot{\delta}_{i}(t)=\Delta \omega_{i}(t)$

$\Delta \dot{\omega}_{i}=-\frac{D_{i}}{2 H_{i}} \Delta \omega_{i}(t)-\frac{\omega_{0}}{2 H_{i}}\left(P_{e i}(t)-P_{m i}\right)$

Generator Electrical Dynamics:

$\dot{E}_{q i}^{\prime}=\left(E_{f i}(t)-E_{q i}(t)\right) \frac{1}{T_{d 0 i}^{\prime}}$

Electrical Equation:

$E_{q i}(t)=E_{q i}^{\prime}(t)-\left(X_{d i}-X_{d i}^{\prime}\right) I_{d i}(t)$

$E_{f i}(t)=k_{c i} u_{f i}(t)$

$P_{e i}(t)=\sum_{j=1}^{n} E_{q i}^{\prime}(t) E_{q j}^{\prime}(t) B_{i j} \sin \left(\delta_{i j}(t)\right)$

$Q_{e i}(t)=-\sum_{j=1}^{n} E_{q i}^{\prime}(t) E_{q j}^{\prime}(t) B_{i j} \cos \left(\delta_{i j}(t)\right)$

$I_{q i}(t)=\sum_{j=1}^{n} E_{q j}^{\prime}(t) B_{i j} \sin \left(\delta_{i j}(t)\right)$

$I_{d i}(t)=-\sum_{j=1}^{n} E_{q j}^{\prime}(t) B_{i j} \cos \left(\delta_{i j}(t)\right)$

$E_{q i}=X_{a d i} I_{f i}(t)$

To alleviate the nonlinearities and interconnections between different generators, direct feedback linearization (DFL) technique is employed. The immeasurable variable $\ddot{E}_{q i}^{\prime}(t)$ is eliminated by differentiating equation (6). Let $\Delta P_{e i}(t)=P_{e i}(t)-P_{m i 0}$ and consider the uncertainty in the generator parameter $T_{d 0 i}^{\prime}$ and $T_{d 0 i}^{\prime}=\bar{T}_{d 0 i}^{\prime}+\Delta T_{d 0 i}^{\prime}$. Choosing the state as $x_{i}^{T}(t)=\left[\begin{array}{lll}\Delta \delta_{i}(t) & \Delta \omega_{i}(t) & \Delta P_{e i}(t)\end{array}\right]$, the characteristic of the generator after linearized by DFL technique is given by the following equations:

$$
\left\{\begin{array}{l}
\Delta \dot{\delta}_{i}(t)=\Delta \omega_{i}(t) \\
\Delta \dot{\omega}_{i}=-\frac{D_{i}}{2 H_{i}} \Delta \omega_{i}(t)-\frac{\omega_{0}}{2 H_{i}}\left(P_{e i}(t)-P_{m i}\right) \\
\Delta P_{e i}(t)=-\frac{1}{T_{d o i}^{\prime}} \Delta P_{e i}(t)+\frac{1}{T_{d o i}^{\prime}} v_{f i}(t) \\
\quad+\sum_{j=1}^{n} E_{q i}^{\prime}(t)\left[\dot{E}_{q j}^{\prime}(t) B_{i j}+E_{q j}^{\prime}(t) \dot{B}_{i j}\right] \sin \left(\delta_{i j}(t)\right) \\
\quad-E_{q i}^{\prime}(t) \sum_{j=1}^{n} E_{q j}^{\prime}(t) B_{i j} \cos \left(\delta_{i j}(t)\right) \Delta \omega_{i j}(t)
\end{array}\right.
$$

where $v_{f i}(t)=k_{c i} I_{q i}(t) u_{f i}(t)+\left(X_{d i}-X_{d i}^{\prime}\right) I_{d i}(t) I_{q i}(t)$

$$
-\bar{T}_{d o i}^{\prime} Q_{e i}(t) \Delta \omega_{i}(t)-P_{m i 0}
$$

$\mu_{i}(t)=\frac{1}{\bar{T}_{d 0 i}^{\prime}+\Delta T_{d 0 i}^{\prime}}-\frac{1}{\bar{T}_{d 0 i}^{\prime}}$ and $\psi_{i}(t)=\left[\frac{1}{\bar{T}_{d 0 i}^{\prime}}+\mu_{i}(t)\right] \Delta T_{d o i}^{\prime} Q_{e i}(t)$.

Remark 1: After the DFL compensation, the generator model is linear with respect to the new input $v_{f i}(t)$ in steady state of multi-machine power system. However, the DFL compensating law still contains nonlinearities due to the effects of remote dynamics of the $j$ th generator on the $i$ th generator, such as switching of transmission lines when a fault occurs in the power system, the system parameter, $B_{i j}(t)$ will change considerably. In moment of switching breakers under large disturbances, the derivative of susceptance $\dot{B}_{i j}(t)$ changes dramatically and it cannot be bounded. However, it remains zero when structure of transmission lines does not change.

\section{Global CONTROL OF MUlTi-MAChINE POWER SYSTEMS}

In order to take into account the abrupt structure changes of power system, global control design is applied to multi-machine power system to enhance transient stability. The configuration of the proposed global control scheme for multi-machine power system is depicted in Fig. 1, in which, the multi-machine power systems are represented by interconnected subsystems with uncertainties and nonlinearities. Then each subsystem is decomposed into four nonlinear partial systems according to switching actions of breakers under a severe disturbance. Four partial systems represent pre-fault steady state, faulty state, fault clearing steady state and post-fault steady state of the subsystem respectively. Then the DFL technique is used to linearize four partial nonlinear models and decentralized robust controllers are designed for the linearized partial models of different states. Finally, the global control law is obtained by integrating the partial controllers from every subsystem with heterogeneous control membership functions.

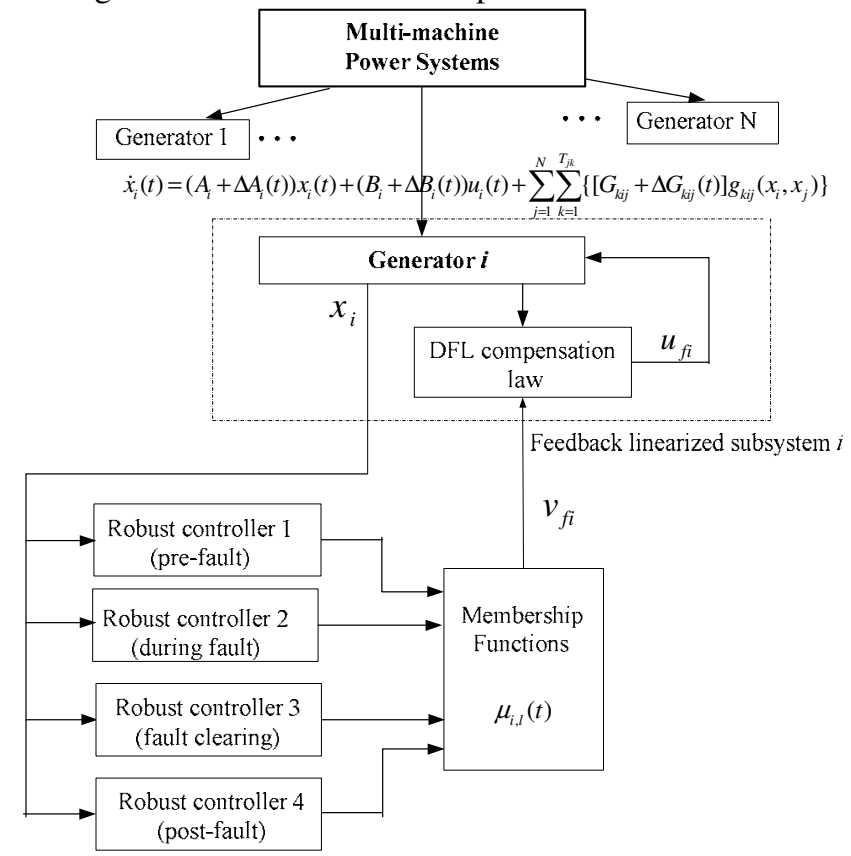

Fig. 1 Global control scheme for multi-machine power system

\section{A. Global control system model for multi-machine power system}

In the global controller design, multi-machine power system is decomposed into pre-fault steady state, faulty state, fault clearing steady states and post-fault steady state. The switching moments were not taken into account since the 
switching time can be neglected during the transient analysis. Therefore, the generalized model of $i$ th subsystems with parameter uncertainties, nonlinearities and interconnections in four states become the following state space form:

$$
\begin{gathered}
\dot{x}_{i}(t)=\left(A_{i, l}+\Delta A_{i, l}(t)\right) x_{i}(t)+\left(B_{i, l}+\Delta B_{i, l}(t)\right) u_{i, l}(t) \\
+\sum_{j=1}^{N} \sum_{k=1}^{T_{j k, l}}\left\{\left[G_{k i j, l}+\Delta G_{k i j, l}(t)\right] g_{k i j, l}\left(x_{i}, x_{j}\right)\right\}
\end{gathered}
$$

where $l$ is the number of partial models for the $i$ th $(i=1,2, \ldots N)$ subsystem; $l=1,2,3$ and $4, i=1,2, \ldots N, k=1, \ldots T_{j k} ; T_{j k}=2$, $u_{i, l}(t) \in \mathfrak{R}^{n}$ is the input variables of the $i$ th subsystem; the matrices $A_{i, l}, B_{i, l}$, and $G_{i j, l}$ are known real constant matrices of appropriate dimension that describe the nominal model of the $l$ th linearized partial models, $\Delta A_{i, l}, \Delta B_{i, l}$, and $\Delta G_{i j, l}$ are real time varying parameter uncertainties of the $l$ th linearized partial models; $g_{k i j, l}\left(x_{i}, x_{j}\right)$ are unknown nonlinear vector functions that represent nonlinearities of the $i$ th subsystem and interactions between the $i$ th subsystem and $j$ th subsystem in the $l$ th linearized partial model.

According to equation (11) and (12), the state variables $x_{i}^{T}(t)=\left[\begin{array}{lll}\Delta \delta_{i}(t) & \Delta \omega_{i}(t) & \Delta P_{e i}(t)\end{array}\right], \quad v_{f i}(t)$ are the input variables,

$A_{i, l}=\left[\begin{array}{ccc}0 & 1 & 0 \\ 0 & -\frac{D_{i}}{2 H_{i}} & -\frac{\omega_{0}}{2 H_{i}} \\ 0 & 0 & -\frac{1}{\bar{T}_{d 0 i}^{\prime}}\end{array}\right] ; B_{i, l}=\left[\begin{array}{c}0 \\ 0 \\ 1 \\ \overline{\bar{T}_{d 0 i}^{\prime}}\end{array}\right] ; \Delta B_{i, l}=\left[\begin{array}{c}0 \\ 0 \\ \mu_{i, l}(t)\end{array}\right] ;$

$\Delta A_{i, l}=\left[\begin{array}{ccc}0 & 0 & 0 \\ 0 & 0 & 0 \\ 0 & -\psi_{i, l}(t) & -\mu_{i, l}(t)\end{array}\right] ; G_{1 i j, l}=G_{2 i j, l}=\left[\begin{array}{l}0 \\ 0 \\ 0\end{array}\right]$;

$\Delta G_{1 i j, l}=\left[\begin{array}{c}0 \\ 0 \\ \gamma_{1 i j, l}(t)\end{array}\right] ; \Delta G_{2 i j, l}=\left[\begin{array}{c}0 \\ 0 \\ \gamma_{2 i j, l}(t)\end{array}\right]$;

$g_{1 i j, l}\left(x_{i}, x_{j}\right)=\sin \left(\delta_{i}(t)-\delta_{j}(t)\right) ; g_{2 i j, l}\left(x_{i}, x_{j}\right)=\Delta \omega_{j}(t) ;$

$\gamma_{1 i j, l}(t)=E_{q i, l}^{\prime}(t)\left[\dot{E}_{q j, l}^{\prime}(t) B_{i j, l}+E_{q j, l}^{\prime}(t) \dot{B}_{i j, l}\right]$;

$\gamma_{2 i j, l}(t)=-E_{q i, l}^{\prime}(t) E_{q j, l}^{\prime}(t) B_{i j, l} \cos \left(\delta_{i j}(t)\right)$.

$\mu_{i, l}(t)=\frac{1}{\bar{T}_{d 0 i}^{\prime}+\Delta T_{d 0 i}^{\prime}}-\frac{1}{\bar{T}_{d 0 i}^{\prime}}$ and $\psi_{i, l}(t)=\left[\frac{1}{\bar{T}_{d 0 i}^{\prime}}+\mu_{i, l}(t)\right] \Delta T_{d o i}^{\prime} Q_{e i, l}(t)$

For the above partial models (13), if the following robust controllers can be designed to guarantee the stability of closed-loop partial systems

$u_{i, l}(t)=-K_{i, l} x_{i}(t)$

Then the control law for the $i$ th subsystem can be obtained by aggregating the partial controllers with heterogeneous control membership functions.

$u_{i}(t)=-\sum_{l=1}^{m_{i}} \mu_{i, l}(t) K_{i, l} x_{i}(t)$ where $\mu_{i, l}(t)$ is the normalized membership function of the linearized partial models and satisfy $\sum_{l=1}^{m_{i}} \mu_{i, l}(t)=1$. The global closed loop system for the $i$ th subsystem is then described by the following equation:

$$
\begin{aligned}
\dot{x}_{i}(t)= & \sum_{l=1}^{m_{i}} \mu_{i, l}(t)\left\{\left(A_{i, l}+\Delta A_{i, l}(t)+\left(B_{i, l}+\Delta B_{i, l}(t) K_{i, l}\right) x_{i}(t)\right.\right. \\
& \left.+\sum_{j=1}^{N} \sum_{k=1}^{T_{j k, l}}\left\{\left[G_{k i j, l}+\Delta G_{k i j, l}(t)\right] g_{k i j, l}\left(x_{i}, x_{j}\right)\right\}\right\}
\end{aligned}
$$

\section{B. Decentralized robust controllers design for partial models}

For the $l$ th linearized partial models of the $i$ th subsystem, the following assumptions can be assumed.

\section{Assumption 1:}

The uncertain matrices $\Delta A_{i, l}, \Delta B_{i, l}$, and $\Delta G_{i j, l}$ are assumed to be of the following structure:
$\left[\Delta A_{i, l}(t) \quad \Delta B_{i, l}(t)\right]=L_{i, l} F_{i, l}(t)\left[\begin{array}{ll}E_{1 i, l} & E_{2 i, l}\end{array}\right]$
$\Delta G_{k i j, l}=L_{k i j, l} F_{k i j, l}(t) E_{k i j, l}$

\section{Assumption 2:}

There exist known matrices $W_{k i, l}$ and $W_{k i j, l}$ such that nonlinear terms $g_{k i j, l}\left(x_{i}, x_{j}\right)$ satisfy:

$\left\|g_{k i j, l}\left(x_{i}, x_{j}\right)\right\| \leq\left\|W_{k i, l} x_{i}(t)\right\|+\left\|W_{k i j, l} x_{j}(t)\right\|$

Remark 2: The ranges of uncertainties in power system model are various with different operating conditions. For example, in the steady states, the uncertainties of the power system only take into account model and system uncertainties, which is a relatively smaller range in comparison with the structure change of power system under a severe disturbance.

To solve the decentralized robust control problem for DFL compensated partial models (13) involves solving the algebraic Riccati equation in [12].

$$
\begin{aligned}
& \left(A_{i, l}-B_{i, l} R_{i, l}{ }^{-1} E_{2 i, l}^{T} E_{1 i, l}\right)^{T} P_{i, l}+P_{i, l}\left(A_{i, l}-B_{i, l} R_{i, l}{ }^{-1} E_{2 i, l}^{T} E_{1 i, l}\right) \\
& \quad+\sum_{j=1}^{N} \sum_{k=1}^{T_{j k}}\left(W_{k j i, l}{ }^{T} W_{k j i, l}+W_{k i, l}{ }^{T} W_{k i, l}\right)+P_{i, l}\left\{L_{i, l} L_{i, l}{ }^{T}-B_{i, l} R_{i, l}{ }^{-1} B_{i, l}{ }^{T}\right. \\
& \quad+\sum_{j=1}^{N} \sum_{k=1}^{T}\left[G_{k i j, l}\left(I-\varepsilon_{k i j, l}{ }^{2} E_{k i j, l}{ }^{T} E_{k i j, l}\right) G_{k i j, l}{ }^{T}\right. \\
& \left.\left.\quad+\frac{1}{\varepsilon_{k i j, l}{ }^{2}} L_{k i j, l} L_{k i j, l}{ }^{T}\right]\right\} P_{i, l}+Q_{i, l}=0
\end{aligned}
$$

where $Q_{i, l}$ are symmetric positive defined matrices, which can be chosen by the designer, $R_{i, l}=E_{2 i, l}^{T} E_{2 i, l}>0, \varepsilon_{k i j, l}$ are scaling parameters to be chosen, with $\varepsilon_{k i j, l}$ satisfying $\varepsilon_{k i j, l}{ }^{2} E_{k i j, l}{ }^{T} E_{k i j, l}<I \quad, \quad \forall i, j=1,2, \ldots . N$. In this case, the stabilizing state-feedback control laws are given by

$u_{i, l}(t)=-R_{i, l}{ }^{-1}\left(B_{i, l}{ }^{T} P_{i, l}+E_{2 i, l}^{T} E_{1 i, l}\right) x_{i}(t)$

\section{Global control using membership functions}

Four partial controllers are separately designed by robust 
control technique for the corresponding partial models: pre-fault, during fault, fault clearing and post-fault system models. Since we consider faults are clear and transmission lines are restored after disturbances, the pre-fault and the post-fault partial models are same. Therefore, three partial models are combined to represent every subsystem in multi-machine systems over the whole operating condition. In order to realize a soft switching type mechanism for changing different operating conditions, the heterogeneous control membership function is used to aggregate all the partial models and construct a global nonlinear model.

In the transient stability study, the rotor speed of the generator, $\Delta \omega_{i}(t)$, the active power of the generator, $\Delta P_{\mathrm{ei}}(t)$, and switching time of the breakers $z$, are used to construct the heterogeneous control membership functions of the partial models $\mu_{i, l}(t)$, where $\gamma_{i}=\sqrt{\Delta \omega_{i}^{2}+\Delta P_{\mathrm{ei}}^{2}}$ and $z$ are considered as state variables. The membership functions of state variables shown in Fig. 2 are as follow:

$$
\begin{aligned}
& \left\{\begin{array}{cc}
m f_{i, 1}\left(r_{i}\right)=\frac{1}{1+e^{-120\left(r_{i}+0.4\right)}}-\frac{1}{1+e^{-120\left(r_{i}-0.4\right)}} & \left|r_{i}\right| \leq 0.4 \\
m f_{i, 2}\left(r_{i}\right)=1-m f_{1}\left(r_{i}\right) & \left|r_{i}\right|>0.4
\end{array}\right. \\
& \left\{\begin{array}{cc}
m f_{3}(z)=\frac{1}{1+e^{-200\left(z-t_{1}\right)}}-\frac{1}{1+e^{-200\left(z-t_{2}\right)}} & t_{1} \leq z \leq t_{2} \\
m f_{4}(z)=1-m f_{3}(z) & z<t_{1} \text { or } \quad z>t_{2}
\end{array}\right.
\end{aligned}
$$

where $t_{1}$ and $t_{2}$ are respectively the opening and closing time of breakers and $t_{1}<t_{2}$.

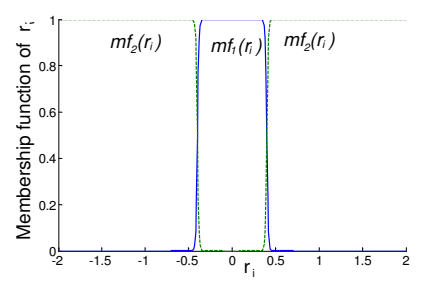

(a) $\gamma_{i}=\sqrt{\Delta \omega_{i}^{2}+\Delta P_{\mathrm{ei}}^{2}}$

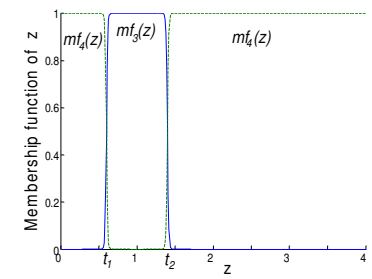

(b) $z$
Fig. 2 The membership functions of state variables $\gamma_{i}$ and $z$

The membership functions of the states variables can be selected as shown in Fig. 2, Then the heterogeneous control membership functions of the partial models $\mu_{i, l}(t)=y_{i, l}(t) / \sum_{l=1}^{3} y_{i, l}(t) \quad$, and where $y_{i, 1}(t)=m f_{i, 1}\left(r_{i}\right) m f_{i, 4}(z)$; $y_{i, 2}(t)=m f_{i, 2}\left(r_{i}\right) m f_{4}(z) ; \quad y_{i, 3}(t)=m f_{3}(z) \quad$ and $\sum_{l=1}^{3} \mu_{i, l}(t)=1$. Therefore, heterogeneous control membership functions for partial models are time-varying according to state variables, $\Delta \omega_{i}(t), \Delta P_{\mathrm{ei}}(t)$ and $\mathrm{z}$. Then the global control law is obtained by aggregating the partial controllers with heterogeneous control membership functions.

$v_{f i}(t)=\sum_{l=1}^{m} \mu_{i, l}(t) v_{f i, l}(t)$
Remark 3: Three robust controllers are designed for the partial subsystems with different operating conditions: pre-fault steady state, faulty state, fault clearing and post-fault steady state. System parameters, fault location and system operating points are all treated as the uncertainties and handled by the decentralized robust control approach in each partial model of subsystems. The global control law is obtained by aggregating the partial controllers with heterogeneous control membership functions.

\section{CASE STUDY}

To evaluate the global control scheme for transient stability enhancement, a three-machine power system shown in Fig. 3 is used in the simulation study [12]. The parameters and physical limits for the example system are given in [12].

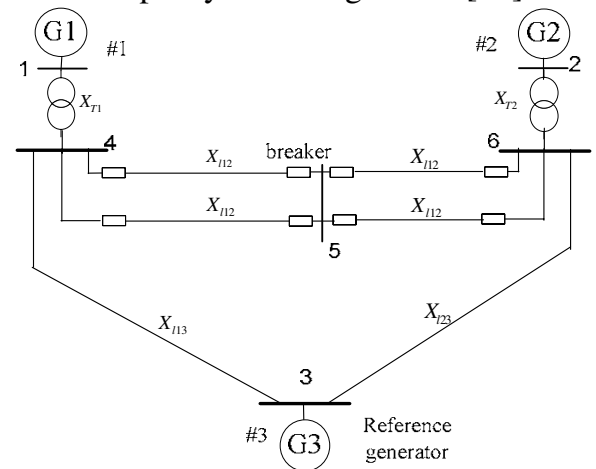

Fig. 3 Three machine example power system

The fault sequences used in the simulation are given as follows:

Stage 1: The system is in a pre-fault steady state.

Stage 2: A three phase short circuit fault occurs at $\mathrm{t}=0.1$ second.

Stage 3: The fault is removed by opening the breaker of the fault line at $t_{1}$ second.

Stage 4: The transmission line is restored with the fault clear at $\mathrm{t}=1.4$ second.

Stage 5: The system is in a post-fault state.

Remark 4: Since the faults are restored at the post-fault state, system structures are same at the pre-fault and post fault stages. Three linearized partial models can be used to represent four operating stages of each generator in the power system.

\section{A. Global controller design}

In the simulation, consider the uncertainties $\Delta T_{d 0 i}^{\prime}=0.1 \bar{T}_{d 0 i}^{\prime}$. The power system model can be written in the form of (13), where $i$ th (1 and 2) subsystems are representative of the generators (1 and 2) in the example system.

For the generator 1, the partial models in the example system is as follow:

$$
\begin{aligned}
\dot{x}_{1}(t)= & \left(A_{1, l}+\Delta A_{1, l}(t)\right) x_{1}(t)+\left(B_{1, l}+\Delta B_{1, l}(t)\right) u_{1, l}(t) \\
& +\Delta G_{112, l} \sin \left(\delta_{1}(t)-\delta_{2}(t)\right)+\Delta G_{212, l}(t) \Delta \omega_{2}(t) \quad l=1,2,3
\end{aligned}
$$

For the generator 2 , the partial models in the example 
system is as follows:

$$
\begin{aligned}
\dot{x}_{2}(t)= & \left(A_{2, l}+\Delta A_{2, l}(t)\right) x_{2}(t)+\left(B_{2, l}+\Delta B_{2, l}(t)\right) u_{2, l}(t) \\
& +\Delta G_{121, l} \sin \left(\delta_{2}(t)-\delta_{1}(t)\right)+\Delta G_{221, l}(t) \Delta \omega_{1}(t) \quad l=1,2,3
\end{aligned}
$$

where $l=1$ represent for the partial model of pre-fault and post-fault steady state. $l=2$ is for faulty state, $l=3$ is for fault clearing steady state. Since the uncertainty bounds only depend on generator parameters $\bar{T}_{d 0 i}^{\prime}, \Delta T_{d 0 i}^{\prime}, Q_{e i, l}(t)$ and $\left|P_{e i, l}(t)\right|_{\max }$, these bounds are very similar for the faulty states and fault clearing steady state. If we assume the same bounds for second and third partial model, the parameters of the second and third partial model are same. Consequently, the same partial controllers are designed for the second and third partial controller. We only present the first and second partial controller design here.

Following the design procedure in Section III and solving the Riccati equation (20), the partial control laws for three partial models of two generators are obtained:

For the first partial model of the generator 1 , choose $\left|Q_{e 1,1}(t)\right|_{\max } \leq 0.6,\left|P_{e 1,1}(t)\right|_{\max } \leq 1.2$, let $Q_{1,1}=\operatorname{diag}[10,1,10]$, $\varepsilon_{112,1}=0.995$ and $\varepsilon_{212,1}=0.889$, solving the algebraic Riccati

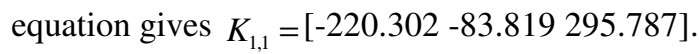

For second and third partial model of the generator 1, choose $\left|Q_{e 1,2}(t)\right|_{\max } \leq 1.5$ and $\left|P_{e 1,2}(t)\right|_{\max } \leq 2.0$, let $Q_{1,2}=\operatorname{diag}[100,1,100]$ and $\varepsilon_{112,2}=0.436, \varepsilon_{212,2}=0.625$, it gives that $K_{1,2}=K_{1,3}=[-753,649-196.026$ 841.759].

The global control law for the generator 1 is then obtained. $u_{1}(t)=-\sum_{l=1}^{3} \mu_{1, l}(t) K_{1, l} x(t)$

For the first partial model of the generator 2, choose $\left|Q_{e 2,1}(t)\right|_{\max } \leq 0.6,\left|P_{e 2,1}(t)\right|_{\max } \leq 1.2$, let $Q_{2,1}=\operatorname{diag}[1,1,1]$, $\varepsilon_{121,1}=0.995$ and $\varepsilon_{221,1}=0.995$, solving the algebraic Riccati equation gives $K_{2,1}=$ [-126.580 -110.519 247.464].

For second partial model of the generator 2, choose $\left|Q_{e 2,2}(t)\right|_{\max } \leq 1.5$ and $\left|P_{e 2,2}(t)\right|_{\max } \leq 2.0$ let $Q_{2,2}=\operatorname{diag}[1,1,1]$ and $\varepsilon_{121,2}=0.3, \quad \varepsilon_{221,2}=0.3$, it gives that $K_{2,2}=K_{2,3}=$ [-299.472 -269.762 893.627].

The global control law for the generator 2 is then obtained. $u_{2}(t)=-\sum_{l=1}^{3} \mu_{2, l}(t) K_{2, l} x(t)$

Remark 5: The decentralized global controllers are designed separately and based on the local measurement only in the interconnected systems. The decentralized feedback laws guarantee the overall stability of multi-machine systems with uncertainties and interconnections.

\section{B. Simulation results}

To evaluate the global control scheme for transient stability enhancement, the three-machine power system shown in Fig.3 is operate upon the following conditions:

$$
\begin{aligned}
& \delta_{10}=60.78^{0} ; P_{e 10}=1.1 \text { p.u.; } V_{t 10}=1.0 \text { p.u., } \\
& \delta_{20}=60.64^{0}, P_{e 20}=1.0 \text { p.u.; } V_{t 20}=1.0 \text { p.u., }
\end{aligned}
$$

A symmetrical three-phase short circuit fault is considered at the terminal of the generator 1 . The performance of global controller for generator excitation is tested by comparing with classical linear controllers. The parameters and physical limits of linear controllers are given in Appendix.

The three-machine power system operates with the fault clearing time $t_{1}=0.22 \mathrm{sec}$.. The comparisons of the power angles of the machines, $\delta_{i j}(t)$, the real power output of machines,$P_{e i}(t)$, and the relative speeds of the machines, $\omega_{i}(t)$, are shown in Fig.4 (a)-(h) respectively.

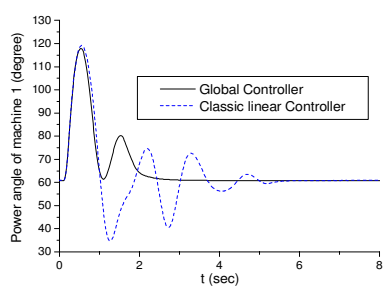

(a) $\delta_{13}(t)$

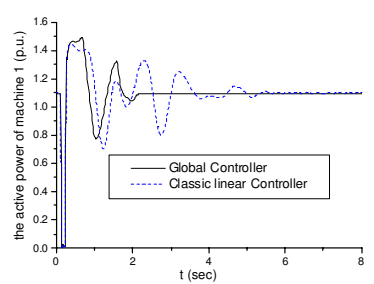

(c) $P_{e 1}(t)$

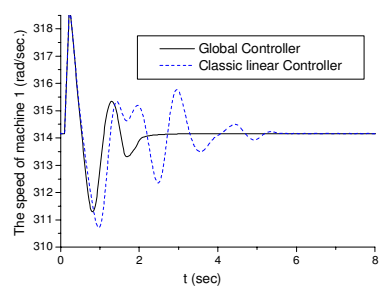

(e) $\omega_{1}(t)$

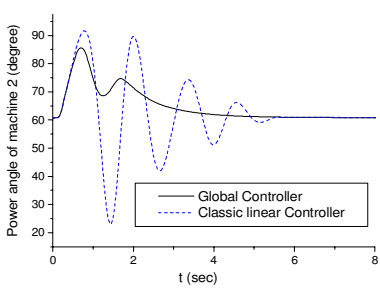

(b) $\delta_{23}(t)$

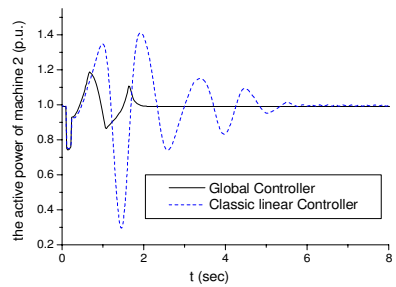

(d) $P_{e 2}(t)$

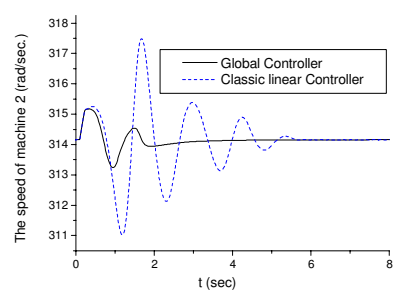

(f) $\omega_{2}(t)$
Fig. 4 Responses of machines with different control schemes

The above simulation results demonstrate that global controller designed greatly enhance transient stability of multi-machine power system in comparison of the classical linear controller. The proposed controller can provide satisfactory global performance in power system transient stability, even under the most severe fault occurred at the terminal of the generator.

Note that different operating conditions, fault locations have been considered to test the robustness of the proposed 
global control design for the three machine power system. The similar conclusions can be drawn from the simulation of those cases, which are not provided in the paper due to length limitation.

\section{CONCLUSIONS}

In this paper, a global control strategy was applied to the multi-machine power system to enhance transient stability. Global control system models are successfully introduced to represent the multi-machine power system by interconnected subsystems. Then each subsystem is decomposed into four nonlinear partial systems according to switching actions of breakers under a severe disturbance. Four partial systems represent pre-fault steady state, faulty state, fault clearing and post-fault steady state of the subsystem respectively. After that, the direct feedback linearization (DFL) technique is used to linearize four partial nonlinear models and decentralized robust controllers are designed for the linearized partial models for different states. All controllers are designed based on locally available measurement. Finally, the global controller is the summation of all the control actions from every subsystem to guarantee the stabilization of the interconnected nonlinear systems.

This global control approach improves the feasibility of multi-machine power system control by introducing decentralized partial controllers, which coordinate different controllers effectively and smoothly by membership functions. The proposed global control scheme is applied to multi-machine power systems. A three machine power system is used as an example to evaluate the global control scheme for power system transient stability enhancement. The simulation results showed that the proposed global controller can rapidly damp the oscillations irrespective of operating conditions and fault locations.

\section{APPENDIX}

\section{A. Parameters of the linear excitation controller}

AVR (IEEE ST1A excitation system):

$K_{A}=60 ; T_{A}=0.05 \mathrm{sec}$.; $K_{f}=0.05 ; T_{f}=1.0 \mathrm{sec}$.; $I_{L R}=4.4$ p.u.;

$K_{L R}=4.54$ p.u.; $\left|V_{A}\right|<999.0$ p.u.; $\left|E_{f}\right|<6.0$ p.u.

PSS:

The transfer function of PSS can be represented as:

$G_{P S S}=K_{P S S} \frac{T_{r} s}{1+T_{r} s} \frac{\left(1+T_{1} s\right)^{2}}{\left(1+T_{2} s\right)^{2}} \cdot K_{P S S}=15 ; T_{r}=3 \mathrm{sec}$; $T_{1}=0.25$
sec.; $T_{2}=0.05 \mathrm{sec} . ;\left|V_{P S S}\right|<0.05$ p.u.

\section{REFERENCE}

[1] F. Zheng, Q. G. Wang and T. H. Lee, "Robust PI controller design for nonlinear system via Fuzzy Modeling Approach", IEEE Transactions on Systems, Man, and Cybernetics Part A: Systems and Humans, Vol. 31, No. 6, pp. 666-675, 2001.
[2] X. Dai, K. Zhang, T. Zhang and X. Lu, "ANN generalized inversion control of turbo-generator governor", IEE Proceedings-Generation, Transmission and Distribution, Vol. 151, No 3, pp. 327 - 333, 2004.

[3] A. Hasanovic and A. Feliachi, "Genetic algorithm based inter-area oscillation damping controller design using MATLAB", IEEE Power Engineering Society Summer Meeting, Chicago, USA Vol. 3, pp. 1136 $-1141,2002$.

[4] H. R. De Azevedo and K. P. Wong, "A fuzzy logic controller for permanent magnet synchronous machine-a sliding mode approach", Power Conversion Conference, Yokohama, Japan, pp. 672 - 677, 1993.

[5] J. W. Chapman, M. D. Ilic, C. A. King, L. Eng and H. Kaufman, "Stabilizing a multi-machine power system via decentralized feedback linearizing excitation control," IEEE Transactions on Power System, Vol. 8, No. 4, pp. 830-839, 1993.

[6] L. Jiang, Q. H. Wu and J. Y. Wen, "Decentralized nonlinear adaptive control for multimachine power systems via high-gain perturbation observer", IEEE Transactions on Circuits and Systems, Vol. 51, No. 10, pp. $2052-2059,2004$.

[7] D. J. Hill, Y. Guo, M. Larsson, and Y. Wang "Global control of complex power systems", in Bifurcation Control: Theory and Applications, Springer-Verlag, 2003.

[8] Y. Guo, D. J. Hill and Y. Wang, "Global transient stability and voltage regulation for power system", IEEE Transactions on Power Systems, Vol. 16, No. 4, pp.678-688, 2001.

[9] Y. Wang, G. H. Zhang and D. J. Hill, "Robust power system transient stability enhancement: a global control approach," the 6th IFAC symposium on Nonlinear Control Systems, Sep. 2004, Stuttgart, Germany.

[10] G. H. Zhang and Y. Wang, "Global Control of Power Systems for Transient Stability and Voltage Regulation", The 15th Power System Computation Conference, August 22-26, 2005, Liege, Belgium.

[11] P. M. Anderson and A. A Fouad, Power system control and stability, (Second Edition), New Jersey, IEEE Press, 2003.

[12] Y. Wang, G. Guo and D. J. Hill, "Robust decentralized nonlinear controller design for multi-machine power systems", Automatic. VOL33. No.9, 1997, pp. 1725-1733. 